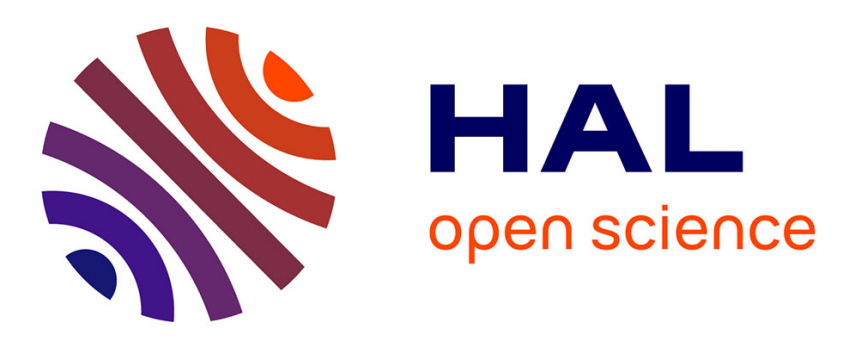

\title{
Reliability estimation measure: Generic Discounting Approach
}

\author{
Ahmed Samet, Eric Lefevre, Imen Hammami, Sadok Ben Yahia
}

\section{To cite this version:}

Ahmed Samet, Eric Lefevre, Imen Hammami, Sadok Ben Yahia. Reliability estimation measure: Generic Discounting Approach. International Journal of Pattern Recognition and Artificial Intelligence, 2015, 29 (07), pp.1559011. 10.1142/S0218001415590119 . hal-03354137

\section{HAL Id: hal-03354137 https://hal.science/hal-03354137}

Submitted on 24 Sep 2021

HAL is a multi-disciplinary open access archive for the deposit and dissemination of scientific research documents, whether they are published or not. The documents may come from teaching and research institutions in France or abroad, or from public or private research centers.
L'archive ouverte pluridisciplinaire HAL, est destinée au dépôt et à la diffusion de documents scientifiques de niveau recherche, publiés ou non, émanant des établissements d'enseignement et de recherche français ou étrangers, des laboratoires publics ou privés. 


\title{
Reliability estimation measure: Generic Discounting Approach
}

\author{
Ahmed Samet ${ }^{1,2}$, Eric Lefèvre ${ }^{1}$, Imen Hammami ${ }^{2}$, Sadok Ben Yahia ${ }^{2,3}$ \\ ${ }^{1}$ Univ. Lille Nord de France UArtois, EA 3926 LGI2A \\ F-62400, Béthune, France \\ firstname.lastname@univ-artois.fr \\ ${ }^{2}$ University of Tunis El Manar, LIPAH Laboratory, Faculty of Sciences of Tunis, Tunisia \\ firstname.lastname@fst.rnu.tn \\ ${ }^{3}$ Institut Mines-TELECOM, TELECOM SudParis, UMR CNRS Samovar, \\ 91011 Evry Cedex, France
}

\begin{abstract}
In the belief function theory, several measures of uncertainty have been introduced. One of their possible use is unreliable source discounting before the fusion stage. Two different measures of uncertainty exist which are the intrinsic and extrinsic ones. The intrinsic measure makes it possible to assess the source's confusion whereas the extrinsic one measures the contradiction between sources. In this paper, we associate both measures in order to estimate the global reliability of a source. This method, named Generic Discounting Approach (GDA), is proposed in two different versions: Weighted GDA and Exponent GDA. Those reliability measures are integrated into a classifier. The method was tested, against to some pioneer approaches, on several UCI datasets as well as on an urban image classification problem and showed very encouraging results.
\end{abstract}

Keywords: Belief function theory; Discounting; Classification; Conflict management; Source confusion.

\section{Introduction}

The belief function theory, introduced $b^{1}{ }^{1}$ and formalized $b^{2}$, has been shown to act as a powerful mathematical background within the information fusion domain as it allows one to express uncertainty and imprecision. In addition to uncertainty handling, this theory allows the extraction of the most likely proposition from multiple sources of provided information. The fusion ability of this formalism is granted by several combination rules; the oldest one is the Dempster rule of combination. However, ${ }^{3}$ has highlighted its counterintuitive behavior. As a result, many works have tackled this conflict management issue proposing different types of solutions, that could be split into two main family approaches: (i) Conflict management approaches based on discounting the unreliable sources ${ }^{4,5,6}$; (ii) Redistribution of the conflict after source's combination ${ }^{7,8}$.

The discounting approach has largely been addressed in the induced literature e.g. ${ }^{8}$. It relies on the fact that a conflict is generated by the unreliability of at least one 


\section{Ahmed Samet et al.}

source. The unreliable sources are then discounted by a coefficient affecting their consideration during the combination phase. Several works have been carried out in this stream in the sake of finding those discounting factors ${ }^{4,5,9}$. However, comparatively to the redistribution family, the discounting approaches are less explored by research because of difficulty of measuring source reliability. Nevertheless, some interesting works have been proposed recently based on source's distance measure, providing some interesting results ${ }^{10,11,12}$. Indeed, all those works were based on the assumption that the more a source is distant from the other ones (i.e., source in contradiction with other ones), the higher its unreliability.

Several works have highlighted that the conflict resulting from the source combination phase is not necessarily the result of their contradiction ${ }^{2,11,13}$. Indeed, the lack of informativity or the high confusion ${ }^{\mathrm{a}}$ of a source could be a sufficient reason for conflict appearance. In literature, several measures were proposed in order to estimate those conflict causes ${ }^{10,12}$. However, even though the literature witnesses a huge number of conflict management approaches, only a little attention was paid to those considering both conflict managements.

In this paper, we distinguish two possible origins of conflicts and we take them into account. The intrinsic conflict caused by the confusion rate of a source to determine certain classes. The second considered conflict origin is the extrinsic conflict which indicates to what extent the obtained sources are in contradiction. To eliminate conflict and enhance the right hypothesis during the fusion process, we have to consider those two conflict causes. To achieve this purpose, two new methods are introduced to estimate the sources reliability, namely the Weighted Generic Discounting Approach (GDA-W) and Exponent Generic Discounting Approach (GDA-E). The proposed discounting approaches were integrated into a based belief function distance classifier ${ }^{14}$. The proposed classifier is experimented within two different contexts. In the first stage, we carried out comparative tests between our classifier and several pioneer approaches on some benchmarks. We also have led tests of our classifier performance in an urban image classification problem.

The remainder of this paper is organized as follows: Basic concepts of belief functions are recalled in Section 2. Without being exhaustive, various measures of intrinsic and extrinsic conflict, developed in the framework of belief functions, are exposed in Section 3. In Section 4, we introduce both proposed variants of the Generic Discounting Approach (GDA) allowing source's reliability estimation. In Section 5, we present a based belief function classifier that integrated the GDA discounting for source fusion improvement. The proposed classifier is experimented on several benchmark datasets as well as on a high resolution urban image classification problem comparatively to some pioneering works. Finally, we conclude and we sketch issues of future work.

${ }^{\mathrm{a}} \mathrm{A}$ source is said to be confused if it is hard to pick a decision from its brought information. 


\section{Belief function theory}

The Belief function theory was initiated by the pioneering work of ${ }^{1}$ on the upper and lower Probabilities. The development of the theory formalism is owed to ${ }^{2}$. Shafer showed the benefits of belief function theory in modelling uncertain knowledge. In addition, it allows to fuse information that was obtained through various sources. The belief function theory is based on several concepts. In this part, we intend to present the main concepts of this theory. For more details, the interested reader may refer to ${ }^{2},{ }^{15}$.

\subsection{Frame of discernment}

The frame of discernment is the set of possible answers for a treated problem and generally noted $\Omega$. It is composed of $N$ exhaustive and exclusive hypotheses:

$$
\Omega=\left\{H_{1}, H_{2}, \ldots, H_{N}\right\}
$$

The exhaustive assumptions indicate that the solution of the problem is necessarily one of the hypotheses $H_{i}$ from the frame of discernment. The exclusivity condition support the unicity of the solution $H_{i} \cap H_{j}=\emptyset, \forall i \neq j$. From the frame of discernment $\Omega$, we deduce the superset $2^{\Omega}$ containing all the $2^{N}$ subsets $\mathrm{A}$ of $\Omega$ :

$$
2^{\Omega}=\{A, A \subseteq \Omega\}=\left\{H_{1}, H_{2}, \ldots, H_{N}, H_{1} \cup H_{2}, \ldots, \Omega\right\}
$$

This set constitutes a reference to assess the veracity of any proposal.

\subsection{Basic Belief Assignment}

The Basic Belief Assignment (BBA) or the basic belief is function $m$ is the mapping from elements of the power set $2^{\Omega}$ into $[0,1]$ so that as:

$$
m: 2^{\Omega} \longrightarrow[0,1]
$$

having as constraints:

$$
\left\{\begin{array}{l}
\sum_{A \subseteq \Omega} m(A)=1 \\
m(\emptyset)=0 .
\end{array}\right.
$$

where $m(A)$ is the confidence strictly assigned to $A$ without is being able to be divided on the hypothesis which composes it. Each subset $A$ of $2^{\Omega}$, fulfilling $m(A)>$ 0 , is called focal element. Constraining $m(\emptyset)=0$ is the normalized form of a BBA and this corresponds to a closed-world assumption ${ }^{16}$, while allowing $m(\emptyset)>0$ corresponds to an open world assumption ${ }^{15}$.

A BBA $m$ is simple if it has no more than two focal sets, $\Omega$ included. A simple $\mathrm{BBA}$ can be represented in this form:

$$
\begin{array}{r}
m(A)=1-w \\
m(\Omega)=w
\end{array}
$$


Ahmed Samet et al.

with $w \in[0,1]$ and $A \subseteq \Omega$. This kind of BBA is denoted as $A^{w}$.

From a BBA, another function can be defined. The plausibility, denoted $P l(A)$, estimates the maximum potential support that could be given to $A$, if further evidence becomes available and is defined by:

$$
P l(A)=\sum_{B \cap A \neq \emptyset} m(B) .
$$

Finally, the commonality function $q$ is defined from $2^{\Omega} \rightarrow[0,1]$ such as:

$$
q(A)=\sum_{B \supseteq A} m(B), \forall A \in \Omega
$$

\subsection{Discounting}

Assuming that a source of information has a reliability rate equal to $(1-\alpha)$ where $(0 \leq \alpha \leq 1)$, such a meta-knowledge can be taken into account using the discounting operation introduced $\mathrm{by}^{2}$, and is defined by:

$$
\left\{\begin{array}{l}
m^{\alpha}(B)=(1-\alpha) \times m(B) \quad \forall B \subset \Omega \\
m^{\alpha}(\Omega)=(1-\alpha) \times m(\Omega)+\alpha
\end{array}\right.
$$

A discount rate $\alpha$ equal to 1 means that the source is not reliable and the piece of information that is provided cannot be taken into account. On the contrary, a null discount rate indicates that the source is fully reliable and the piece of information that is provided is entirely acceptable. Thanks to discounting, an unreliable source's BBA is transformed into a function assigning a larger mass to $\Omega$.

\subsection{Combination rules}

The combination rules are used to combine several belief functions provided by different sources in order to synthesize a single BBA. In this subsection, we survey some pioneer combination rules.

\subsubsection{Conjunctive rule}

The belief function theory makes it possible to combine some information modelled as BBA. Several operators were defined such as the conjunctive rule. This combination operator assigns the mass to propositions initially confirmed by the sources. For two sources $S_{1}$ and $S_{2}$ having respectively $m_{1}$ and $m_{2}$ as BBA, the conjunctive rule is defined by:

$$
m_{\circledast}=m_{1} @ m_{2} .
$$

For an event $A, m_{\circledast}$ can be written as follows:

$$
m_{\oplus}(A)=\sum_{B \cap C=A} m_{1}(B) \times m_{2}(C) \quad \forall A \subseteq \Omega .
$$


However, the conjunctive combination result does not fulfil the closed-world condition since it gives a conflictual mass.

\subsubsection{Dempster's rule of combination}

The normalized version of conjunctive rule, proposed by ${ }^{1}$, integrates a conflict management approach that redistributes the generated conflictual mass. The Dempster rule of combination, so called orthogonal sum, is defined as follows:

$$
m_{\oplus}=m_{1} \oplus m_{2} .
$$

For two sources $S_{1}$ and $S_{2}$, the aggregation of evidence can be written as follows:

$m_{\oplus}(A)=\frac{1}{1-m(\emptyset)} \sum_{B \cap C=A} m_{1}(B) \times m_{2}(C)=\frac{1}{1-m(\emptyset)} m_{\circledast}(A) \quad \forall A \subseteq \Omega, A \neq \emptyset$.

where $m(\emptyset)$ is defined by:

$$
m(\emptyset)=\sum_{B \cap C=\emptyset} m_{1}(B) \times m_{2}(C)=m_{\circledast}(\emptyset) .
$$

$m(\emptyset)$ represents the conflict mass between $m_{1}$ and $m_{2}$.

\subsubsection{Cautious conjunctive rule}

Another combination rule, called the cautious conjunctive rule ${ }^{17}$, was proposed in case of the independence of the sources is compromised. Let $m_{1}$ and $m_{2}$ be two non dogmatic mass functions defined on the frame of discernment $\Omega$. The cautious conjunctive combination of $m_{1}$ and $m_{2}$, denoted by $m_{1} \bigotimes_{2}=m_{1} @ m_{2}$, is defined on the basis of a weight function below:

$$
w_{1} \bowtie 2(A)=w_{1}(A) \wedge w_{2}(A), \forall A \subset \Omega
$$

where $\wedge$ is the minimum operator. Then, $m_{1} \otimes_{2}$ is computed as follows:

$$
m_{1} \otimes_{2}=\oplus_{A \subset \Omega} A^{w_{1}(A) \wedge w_{2}(A)}
$$

where the weight $w(A)$ can be obtained from the commonalities by the following formula:

$$
w(A)=\prod_{B \supseteq A} q(B)^{(-1)^{|B|-|A|+1}}
$$

\subsection{Pignistic probability}

In the literature, we often come a cross the notion of pignistic probability. The pignistic probability, denoted BetP, was proposed by ${ }^{18}$ within its Transferable Belief Model (TBM) approach. TBM is based on the differentiation between the knowledge representation and decision-making level. In the decision phase, the pignistic 
transformation consists in distributing equiprobably the mass of a proposition $A$ on its included hypotheses. Formally, the pignistic probability is defined by:

$$
\operatorname{Bet} P\left(H_{n}\right)=\sum_{A \subseteq \Omega} \frac{\left|H_{n} \cap A\right|}{|A|} \times m(A) \quad \forall H_{n} \in \Omega .
$$

\section{Conflict measures}

Within the framework of the belief function theory, the measure $m(\emptyset)$ (equation 11) is often used as the only measure to quantify the conflict. However, it is not satisfactory because it does not consider all conflictual situations ${ }^{2}$. Recently, several works have proposed other measures ${ }^{13,19,20,21}$. In this section, several conflict measures (or discordance measures) developed within the framework of belief functions are presented. These measures can be classified into two categories:

- The measures which allow the evaluation of the extrinsic conflict (discordance between two bodies of evidence) and will be labelled extrinsic measures.

- The measures which allow the estimation of the intrinsic conflict (confusion rate of a source) and which will be called intrinsic measures.

\subsection{Extrinsic conflict}

Several measures of extrinsic conflict have been studied in order to model the disagreement between sources. Indeed, if one source opinion disagrees with another, then their fusion will lead to an important conflictual mass ${ }^{22}$. An extension of the Euclidean distance is given by ${ }^{23} \cdot \mathrm{In}^{24}$, Tessem introduced a distance measure between the pignistic probabilities that are associated to mass functions. Other distances were studied to define distance between two BBAs as the sum of differences of conflicting normalized plausibility masses ${ }^{11}$. Some authors have directly defined distance between different mass functions such as ${ }^{10}$ that has the advantage of taking into account the cardinality of focal elements. This distance complies with the metric axioms and is an appropriate measure of the contradiction between two BBAs. In the remainder, we have chosen it to measure the contradiction between two BBAs and it is formalized as:

$$
d\left(m_{1}, m_{2}\right)=\sqrt{\frac{1}{2}\left(m_{1}-m_{2}\right)^{t} \cdot D \cdot\left(m_{1}-m_{2}\right)}
$$

where:

$$
D(A, B)=\left\{\begin{array}{l}
1 \text { if } A=B=\emptyset \\
\frac{|A \cap B|}{|A \cup B|} \text { if } A, B \subseteq 2^{\Omega} .
\end{array}\right.
$$

For further details, the interested reader may refer to ${ }^{25}$.

The use of the distance such as a conflict measure is criticized ${ }^{21,20,26} . \mathrm{In}^{21}$, the authors proposed several properties that a conflict measure should sustain. Indeed, 
Destercke and Burger highlighted that a distance can not be used straightforward as a conflict measure until it satisfies the following properties ${ }^{21}$ :

- Extreme conflict values: the maximal distances should be obtained in case of total conflict.

- Insensitivity to refinement: the distance measures should not be sensitive to the frame $\Omega$ size.

- Imprecision monotonicity: for two BBAs $m_{1}$ and $m_{2}$ with distance $d\left(m_{1}, m_{2}\right)=0$. Then, for $m_{1} \sqsubset_{s} m_{1}^{\prime}{ }^{\mathrm{b}}, d\left(m_{1}^{\prime}, m_{2}\right)$ must be equal to 0 .

- Ignorance is bliss: a vacuous BBA should have a null distance from any nonvacuous one.

- BBA extension: Extending distances to more than two masses is not straightforward, and existing proposals ${ }^{5}$ usually come back to pairwise distances by some means.

$\mathrm{In}^{21}$, the authors proposed a conflict measure which satisfies these properties. In addition, an extrinsic measure of conflict was proposed and called external conflict. The external conflict $E C\left(m_{1}, m_{2}\right)$ between two sources $S_{1}$ and $S_{2}$ is computed as follows:

$$
E C\left(m_{1}, m_{2}\right)=1-\max _{\omega \in \Omega} \min \left(P l_{1}^{\prime}(\omega), P l_{2}^{\prime}(\omega)\right)
$$

where $P l_{i}^{\prime}(\omega)$ is the normalized contour plausibility found as follows:

$$
P l_{i}^{\prime}=\frac{P l_{i}}{\max _{\omega \in \Omega} P l_{i}(\omega)} .
$$

\subsection{Intrinsic measures}

The intrinsic conflict quantifies the consistency between the different focal elements inside the BBA. Several measures have been proposed in the literature, that take into account the inclusion relations between the focal elements present in the BBA. Several intrinsic measures were proposed such as the confusion distance introduced $\mathrm{by}^{28} \cdot \mathrm{In}^{5}$, the authors define the notion of auto-conflict (initially introduced by ${ }^{29}$ ) given by the weight assigned to the emptyset generated by the conjunctive combination between $m$ and itself. Nevertheless, the auto-conflict is a kind of confusion measure that depends on the number of combination made. $\mathrm{In}^{12}$, the authors introduced a contradiction measure that no longer depends on order that can be written as follows:

$$
\operatorname{contr}(m)=c \sum_{X \subseteq 2^{\Omega}} m(X) \cdot D\left(m, m_{X}\right)
$$

\footnotetext{
${ }^{\mathrm{b}} m_{1}$ is a specialization of $m_{2}$ (noted $m_{1} \sqsubset_{s} m_{2}$ ) if $m_{1}$ can be obtained from $m_{2}$ by distributing each mass $m_{2}(B)$ to subsets of $B$ as follows $m_{1}(A)=\sum_{B \subseteq \Omega} S(A, B) m_{2}(B), \forall A \subseteq \Omega$. $S(A, B)$ is the specialization matrix indicating the proportion of $m_{2}(\bar{B})$ transferred to $A \subseteq B^{27}$.
} 
where $m_{X}(X)=1, X \in 2^{\Omega}$ is the categorical ${ }^{c}$ BBA, $c$ is normalization constant and $D$ is the ${ }^{10}$ 's distance (see subsection 3.1). Another approach was presented by ${ }^{11}$ using the normalized plausibility transformation to measure the internal conflict given by:

$$
P l_{-} \operatorname{Int} C(m)=1-\max _{\omega \in \Omega} P l(\omega) .
$$

Starting from this definition, there are many BBA without any internal conflicts: all BBA having $X \subseteq \Omega, P l(X)=1$. There are some examples of BBA having no internal conflict: categorical, simple support, consonant BBA ${ }^{\mathrm{d}}$. Finally all BBA, whose all focal elements have non-empty intersection, have no internal conflict. Interesting enough, The internal conflict was also presented as a measure for the conflict resulting from the confusion of a source ${ }^{21}$.

\subsection{Conflict measures and discounting}

Several works have been carried out to discount the BBAs ${ }^{30,31}$. However, these studies are usually based on a learning database. Very few studies used measures of conflict to adapt the belief functions and in this case the used measures are extrinsic measures of conflict.

The first use of extrinsic measure to discount the belief functions was performed by $^{32}$. Initially, in this approach, a similarity matrix is build between belief functions. Next, the values of this matrix is used to weight the BBAs. This approach has been modified by choosing a new similarity matrix ${ }^{33}$.

${ }^{5}$ propose using a function that quantifies the conflict between BBA. This function, called $\operatorname{Conf}(.,$.$) , is defined as:$

$$
\operatorname{Conf}(i, E)=\frac{1}{M-1} \sum_{k=1 ; i \neq k}^{M} \operatorname{Conf}(i, k)
$$

with $M$ is the number of belief functions produced respectively by $M$ sources called $S_{1}, \ldots, S_{M}$ and $E$ is the set of BBAs such that $\left\{m_{k} \mid k=1, \ldots, M\right.$ and $\left.k \neq i\right\}$. The function $\operatorname{Conf}(i, k)$ is obtained using a BBA distance introduced by Jousselme (equation 16):

$$
\operatorname{Conf}(i, k)=d\left(m_{i}, m_{k}\right) .
$$

The value $\operatorname{Conf}(i, E)$ quantifies the average conflict between the BBA $m_{i}$ and the BBAs of the set $E$. Another proposition consists in comparing the BBA $m_{i}$ with the BBA of the artificial expert representing the combined opinions of all the experts in $E$. The measure Conf can be obtained by:

$$
\operatorname{Conf}(i, E)=d\left(m_{i}, m_{*}\right)
$$

\footnotetext{
${ }^{\mathrm{c}} \mathrm{A}$ BBA with only one focal element $A$ is said to be categorical and is denoted $m(A)=1$.

${ }^{\mathrm{d}} \mathrm{A}$ BBA is said consonant if its focal elements are nested.
} 


\begin{tabular}{|c|c|c|c|c|c|c|c|c|c|c|}
\hline \multirow{2}{*}{\multicolumn{2}{|c|}{$m_{1}$}} & \multirow[b]{2}{*}{$m_{2}$} & \multirow[b]{2}{*}{$m_{3}$} & \multicolumn{2}{|c|}{ discounting } & \multirow[b]{2}{*}{$m_{1}$} & \multirow[b]{2}{*}{$m_{2}$} & \multirow[b]{2}{*}{$m_{3}$} & \multicolumn{2}{|c|}{ discounting } \\
\hline & & & & (1) & $\begin{array}{l}+ \\
\text { (1) }\end{array}$ & & & & (1) & $\begin{array}{l}+ \\
\text { (1) }\end{array}$ \\
\hline 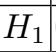 & 0.230 & 0.200 & 0.800 & \begin{tabular}{|l|}
0.151 \\
\end{tabular} & 0.182 & \begin{tabular}{|l|l|}
$H_{1}$ & 0.450 \\
\end{tabular} & 0.400 & 0.470 & 0.156 & 0.158 \\
\hline $\mathrm{H}_{2}$ & 0.570 & 0.600 & 0.100 & \begin{tabular}{|l|}
0.119 \\
\end{tabular} & 0.250 & \begin{tabular}{|l|l|}
$\mathrm{H}_{2}$ & 0.450 \\
\end{tabular} & 0.500 & 0.430 & 0.174 & 0.177 \\
\hline$\Omega$ & 0.200 & 0.200 & 0.100 & \begin{tabular}{|l|}
0.004 \\
\end{tabular} & 0.028 & \begin{tabular}{l|l|}
$\Omega$ & 0.100 \\
\end{tabular} & 0.100 & 0.100 & 0.001 & 0.001 \\
\hline & 0.000 & 0.000 & 0.000 & 0.726 & 0.540 & \begin{tabular}{l|l|}
$\emptyset$ & 0.000
\end{tabular} & 0.000 & 0.000 & 0.669 & 0.664 \\
\hline
\end{tabular}

(a)

(b)

Table 1. Extrinsic conflict measure and discounting.

with $m_{*}$ denoting combination of all BBAs of $E$. $m_{*}$ can be obtained by using different combination rules and more precisely the conjunctive rule (equation 8). Once the conflict measure is obtained, the authors have proposed to compute reliability rates as follows:

$$
\beta_{i}=f(\operatorname{Conf}(i, E))
$$

where $f$ is a decreasing function. The authors propose to use the function $f$ defined as follows:

$$
\beta_{i}=\left(1-\operatorname{Conf}(i, M)^{\lambda}\right)^{1 / \lambda}
$$

with $\lambda>0$. The authors in $^{5}$ recommend using $\lambda=1.5$. Extensions of this work use the idea of sequential discount to manage the conflict when combining belief functions ${ }^{4,34}$. Schubert in ${ }^{6}$ uses the idea of sequential discount but the author employs the degree of falsity instead of the distance measure.

\section{Intrinsic and Extrinsic based discounting factors}

In the previous section, different measures were presented allowing the estimation of the two types of conflict. In spite of these measures, to our knowledge there is no reliability estimation method that links both types of conflict measure (see Section 3.3).

Example 1. The example, proposed in Table 1, presents the interest to use these two types of measures simultaneously. Initially in this example (Table 1-a), we study the fusion result of three involved sources but $m_{3}$ is inconsistent with the other ones. The discounting, based only on the extrinsic measure (equation 22), provides good result since the conflict decreases. In the second part of this table (Table 1-b), the sources are now considered undecided. In this case, even through the discounting is based on an extrinsic measure, the conflict remains high.

The aim of the proposed approach is to anticipate the generation of a high conflictual source after the fusion phase. The discounting factors should be found by taking into consideration the ambiguity of each source (intrinsic measure) and 
the distance separating them (extrinsic measure). To achieve this purpose, we based our discounting approach on two distance measures criteria.

In this section, we propose two different discounting approaches that aim to discard the contradictory and non reliable sources that may eventually lead to an important conflict in the resulting fusion BBA. Let us assume the frame of discernment $\Omega$ containing all possible answers for a question $Q$ relatively to the sources $S_{1}, \ldots, S_{M}$. In the fusion stage, to each processed BBA, a new discounting factor is assigned indicating its relevance and its global reliability. Those discounting factors must fulfil the axioms below.

Axiom 1. Let us assume an intrinsic measure $\delta$ and an extrinsic measure $\beta$. A new discounting measure $f(\delta, \beta)$ must fulfil the following axioms:

- $\beta$ must satisfy extreme conflict values, insensitivity to refinement, imprecision monotonicity, ignorance is bliss and BBA extension properties described in subsection 3.1.

- $f$ is an increasing function from $[0,1]^{2} \rightarrow[0,1]$

- $f(1,1)=1$ and $f(0,0)=0$.

Several functions associating an intrinsic and extrinsic measures could satisfy the aforementioned axioms. In this work, we have retained two functions that we denoted as GDA-W and GDA-E.

\subsection{Weighted Generic Discounting Approach (GDA-W)}

The GDA-W relies on a function $f$ that gathers both aforementioned conflict measures. The function $f$ can be written as follows:

$$
(\delta, \beta) \rightarrow \frac{k . \delta+l . \beta}{k+l}
$$

where $k>0$ and $l>0$ are the weight factors allowing the user to favour one distance measure rather than the other. In equation $27, \delta$ denotes the internal conflict measure of the treated source indicating its confusion rate and $\beta$ the disagreement between the treated source $S_{i}$ and $S_{j}$ with $j \in[1, \ldots, i-1, i+1, \ldots, M]$. To do that, we use the external conflict defined in subsection 3.1. Nevertheless, other Intrinsic and Extrinsic distances variants could be used. So, the values $\delta$ and $\beta$ can be defined by:

$$
\begin{aligned}
& \delta=P l_{-} \operatorname{Int} C\left(m_{i}\right) \\
& \beta=e c\left(m_{i}\right)=E C\left(m_{1}, \ldots, m_{i}, \ldots, m_{M}\right)-E C\left(m_{1}, \ldots, m_{i-1}, m_{i+1}, m_{M}\right)
\end{aligned}
$$

where $e c\left(m_{i}\right)$ is the external conflict between the source $S_{i}$ and the others. $E C\left(m_{1}, \ldots, m_{M}\right)$ is the external conflict for a set of BBAs and is computed as follows:

$$
E C\left(m_{1}, \ldots, m_{M}\right)=1-\max _{\omega \in \Omega} \min _{i=1, \ldots, M}\left\{P l_{i}^{\prime}(\omega)\right\}
$$


Thus, the function $f$ can be written as follows:

$$
(\delta, \beta) \rightarrow \frac{k . P l_{-} \operatorname{Int} C\left(m_{i}\right)+\operatorname{lec}\left(m_{i}\right)}{k+l} .
$$

The classical discounting can be written as follows:

$$
\begin{cases}m^{G D A-W}(B)=(1-f(\delta, \beta)) \times m(B) & \forall B \subset \Omega \\ m^{G D A-W}(\Omega)=(1-f(\delta, \beta)) \times m(\Omega)+f(\delta, \beta) . & \end{cases}
$$

The determination of the weight factors $k$ and $l$ can be found automatically by minimizing the following measures:

$$
\left\{\begin{array}{l}
k>0 \\
l>0 \\
E_{b e t}(k, l)=\sum_{i=1}^{I} \sum_{n=1}^{N}\left(\operatorname{Bet}^{(i)}\left(H_{n}\right)-U_{n}^{i}\right)^{2}
\end{array}\right.
$$

where $B e t P^{(i)}$ represents the pignistic probability of $x^{i}$ vector from the learning base and $U_{n}^{i}$ represents the $x^{i}$ membership.

\subsection{Exponent Generic Discounting Approach (GDA-E)}

The GDA-E is also a discounting approach that estimates source's reliability based on both conflict origins. The GDA-E relies on a function $g$ that can be written as follows:

$$
(\delta, \beta) \rightarrow \beta^{(1-\delta)}
$$

where $\beta$ and $\delta$ are respectively the extrinsic measure and intrinsic measure defined, respectively, in equation 29 and $28 . \mathrm{In}^{35}$, we proposed a different association between extrinsic and intrinsic measures such as the contradiction (see equation 20). The function $g$ can be written as follows:

$$
(\delta, \beta) \rightarrow\left(e c\left(m_{i}\right)\right)^{\left(1-P l_{-} I n t C\left(m_{i}\right)\right)}
$$

Thus, the discounting can be written as follows:

$$
\begin{cases}m^{G D A-E}(B)=(1-g(\delta, \beta)) \times m(B) & \forall B \subseteq \Omega \\ m^{G D A-E}(\Omega) & =(1-g(\delta, \beta)) \times m(\Omega)+g(\delta, \beta)\end{cases}
$$

Table 2 shows the discounting value that could be associated to a BBA for different confusion and distance rates.

Remark 1. The GDA-E measure satisfies all properties of a conflict measure stipulated in subsection 3.1. 
Table 2. The GDA-E discounting value for several cases

\begin{tabular}{|l|c|c|}
\hline Source & With Confusion $\delta=1$ & Without Confusion $\delta=0$ \\
\hline Distant $\beta=1$ & $g(\delta, \beta)=1$ & $g(\delta, \beta)=1$ \\
$\beta \in] 0,1[$ & $g(\delta, \beta)=1$ & $g(\delta, \beta)=e c(m)$ \\
Near $\beta=0$ & $g(\delta, \beta)=1$ & $g(\delta, \beta)=0$ \\
\hline
\end{tabular}

Remark 2. Once discounted with GDA-E or GDA-W, the information sources, represented with BBAs, are no longer considered as independent. Therefore, the combination of the discounted sources should be operated by the cautious conjunctive rule.

Example 2. Let's consider the frame of discernment $\Omega=\left\{H_{1}, H_{2}\right\}$ and three sources $S_{1}, S_{2}$ and $S_{3}$. The belief function values associated to those sources and their discounting values are computed in Table 3.

Table 3. Evaluation of discounting approach on an example

\begin{tabular}{|l|r|r|r|r|r|r|}
\cline { 2 - 7 } & $S_{1}$ & $S_{2}$ & $S_{3}$ & $m_{\circledast}$ & $m_{\AA}^{\text {GDA-E }}$ & $m_{\AA}^{\text {GDA-W }}$ \\
\hline$H_{1}$ & 0.300 & 0.000 & 0.500 & 0.088 & 0.214 & 0.146 \\
\hline$H_{2}$ & 0.300 & 0.800 & 0.200 & 0.326 & 0.286 & 0.380 \\
\hline$\Omega$ & 0.400 & 0.200 & 0.300 & 0.024 & 0.286 & 0.204 \\
\hline$\emptyset$ & 0.000 & 0.000 & 0.000 & 0.562 & 0.214 & 0.270 \\
\hline Intrinsic conflict: $\delta$ & 0.300 & 0.000 & 0.200 & - & - & - \\
\hline Extrinsic conflict: $\beta$ & 0.000 & 0.375 & 0.375 & - & - & - \\
\hline GDA-E: $g(\delta, \beta)$ & 0.000 & 0.375 & 0.456 & - & - & - \\
\hline GDA-W: $f(\delta, \beta)(k=l=1)$ & 0.150 & 0.187 & 0.287 & - & - & - \\
\hline
\end{tabular}

As sketched by the statistics of Table 3, both GDA-W and GDA-E consider $S_{2}$ as a reliable source despite being distant from $S_{1}$ and $S_{3}$ (a classical discounting approach would reject $S_{2}$ for being distant). Thanks to its simple support construction making it without any intrinsic conflict, $S_{2}$ is considered as a reliable source. The same explanation can be applied to $S_{1}$ and $S_{3}$ where despite being close, neither of them can reinforce significantly any hypothesis. Even for GDA-E approach, the BBA's external conflict (extrinsic measure) is powered by the confusion (intrinsic measure) that is why the GDA-E factor is equal to the extrinsic measure when the source is not confused (source $S_{2}$ ). However, it increases as far as the confusion of the source is increased. As it is shown in Table 3, GDA-W and GDA-E have drastically decreased the conflict comparatively to the conjunctive sum, it falls from 0.562 to respectively 0.214 and 0.270 .

Remark 3. Equation 27 and 34 refer to two proposals for estimating the sources 

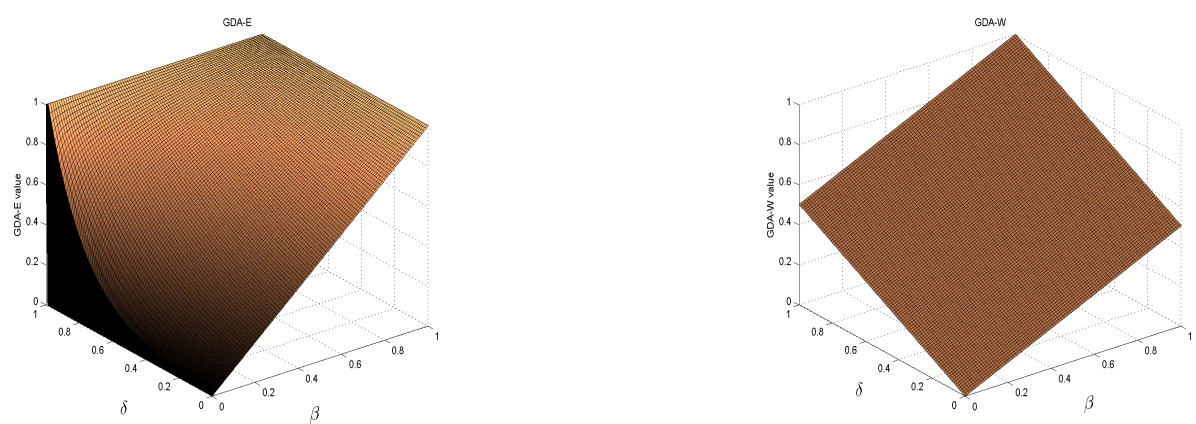

Fig. 1. Comparison between GDA-E and GDA-W behaviour according to $\beta$ and $\delta$.

reliability. Each one relies on an extrinsic and intrinsic measure. However, both are different. GDA-W is a linear formulation of the reliability measure. Indeed, this function calculates the mean of the intrinsic and the extrinsic measure in the case $k=l=1$. Therefore, even if one of the computed measure is equal to 1 , the GDA-W can not reach 1 . In addition, the user can also specify the weight of each measure in case of an a priori information about the nature of the sources (confused, categoric, etc). GDA-E is totally different. It does not require any information about the importance of any measure. Indeed, as highlighted in Figure 1, for the GDA-E function the source becomes more unreliable when the confusion arise. In fact, the GDA-E have the particularity to give 1 in case of one of the used metric is equal to 1 .

Table 4 details the behaviour of both GDA_E and GDA_W with regards to the confusion and the contradiction level. Four BBAs, in the frame of discernment $\Omega=\left\{H_{1}, H_{2}\right\}$, are considered:

$$
\left\{\begin{array} { l } 
{ m _ { 1 } ( \{ H _ { 1 } \} ) = 0 . 9 } \\
{ m _ { 1 } ( \{ H _ { 2 } \} ) = 0 } \\
{ m _ { 1 } ( \Omega ) = 0 . 1 }
\end{array} \quad \left\{\begin{array} { l } 
{ m _ { 2 } ( \{ H _ { 1 } \} ) = 0 . 4 } \\
{ m _ { 2 } ( \{ H _ { 2 } \} ) = 0 . 2 } \\
{ m _ { 2 } ( \Omega ) = 0 . 4 }
\end{array} \quad \left\{\begin{array} { l } 
{ m _ { 3 } ( \{ H _ { 1 } \} ) = 0 . 2 } \\
{ m _ { 3 } ( \{ H _ { 2 } \} ) = 0 . 4 } \\
{ m _ { 3 } ( \Omega ) = 0 . 4 }
\end{array} \quad \left\{\begin{array}{l}
m_{4}\left(\left\{H_{1}\right\}\right)=0 \\
m_{4}\left(\left\{H_{2}\right\}\right)=0.9 \\
m_{4}(\Omega)=0.1
\end{array}\right.\right.\right.\right.
$$

$m_{1}$ is a certain BBA sustaining a hypothesis and $m_{4}$ is its opposite. Both $m_{2}$ and $m_{3}$ are confused BBAs but sustain different focal elements. The symbols $(+)$ and $(-)$ in Table 4, represent respectively the presence and the absence of contradiction (resp. confusion) within a single source.

In case of certain BBAs (rows 1 and 4 of Table 4), both GDA_W and GDA_E have the same behaviour as an extrinsic measure. In other words, when the BBAs are certain, the result of the GDA_E is equal to those of the external conflict $(e c()$.$) .$ In this case, the result of the GDA_W is a weighting of the external conflict. The results become different in case of confusion appearance within the sources (rows 2 and 3 of Table 4). In fact, in case of high confusion with a source, no matter how 
Table 4. Behaviour of the GDA_W and GDA_E with regards to the confusion and the contradiction level

\begin{tabular}{|l|c|c|c|c|c|}
\hline Cases & Contradictory & Confused & $(\mathrm{ec}()$, Pl_IntC(.)) & GDA_E & GDA_W \\
\hline$\left\{m_{1}, m_{1}, m_{1}\right\}$ & $\{-,-,-\}$ & $\{-,-,-\}$ & $\{(0,0),(0,0),(0,0)\}$ & $\{0,0,0\}$ & $\{0,0,0\}$ \\
\hline$\left\{m_{1}, m_{1}, m_{2}\right\}$ & $\{-,-,-\}$ & $\{-,-,+\}$ & $\{(0,0),(0,0),(0,0.2)\}$ & $\{0,0,0\}$ & $\{0,0,0.1\}$ \\
\hline$\left\{m_{1}, m_{1}, m_{3}\right\}$ & $\{-,-,+\}$ & $\{-,-,+\}$ & $\{(0,0),(0,0),(0.25,0.2)\}$ & $\{0,0,0.33\}$ & $\{0,0,0.23\}$ \\
\hline$\left\{m_{1}, m_{1}, m_{4}\right\}$ & $\{-,-,+\}$ & $\{-,-,-\}$ & $\{(0,0),(0,0),(0.9,0)\}$ & $\{0,0,0.9\}$ & $\{0,0,0.45\}$ \\
\hline
\end{tabular}

certain it is, it remains unreliable for GDA_E. The GDA_W is a weighted mean of the extrinsic and intrinsic measures. In this example where the weight factors are chosen equally, the confusion have the same importance as the contradiction. In fact, GDA_W considers the source as unreliable only if its simultaneously confused and contradict the other ones.

In the following section, the GDA discounting approaches are integrated into a belief based classifier.

\section{GDA Classifier and complexity}

In this section, we introduce the based belief function theory GDA classifier. The proposed classifier relies on multi-source fusion and integrates the GDA discounting approach for unreliable source detection. Figure 2 represents the proposed architecture for GDA classifier. The first part of the figure represents the belief function estimation model. The GDA classifier part operates a discounting phase based on extrinsic and intrinsic conflict measures, source fusion and decision. For the first part of this architecture, several belief based classifiers exist, with whom we can obtain belief function estimation such as the likelihood ${ }^{2}$ and the tree based classifiers ${ }^{36}$. In our case, we built the GDA classifier on the distance classifier ${ }^{14}$ for its simplicity and combinatorial explosion avoidance. The properties of this classifier is detailed and compared in $^{37}$.

\subsection{Distance Classifier}

Introduced by ${ }^{14}$, the Distance Classifier (DC) is a based belief function theory and multi-level fusion classification approach. It relies on a learning base in which we store the patterns $x_{i}$ belonging to $H_{n}^{i}$ class. Considering a vector $x$ to classify, the application of the $K$ Nearest Neighbours $(K N N)$ algorithm on the learning base, provides $k$ pieces of evidence. Indeed, each vector $x_{i}$, sufficiently close to $x$ following a distance $d$ brings information about $x$ membership to $H_{n}^{i}$. This information is represented by a BBA $m$ over the set $\Omega$ of classes. A fraction of the unit mass is assigned by $m$ to the singleton $H_{n}^{i}$, and the rest is assigned to the whole frame of discernment $\Omega$. The mass assigned to $m\left(\left\{H_{n}^{i}\right\}\right)$ follows a decreasing function in distance $d$. For each neighbour $x_{i}$ a BBA is modelled as follows: 


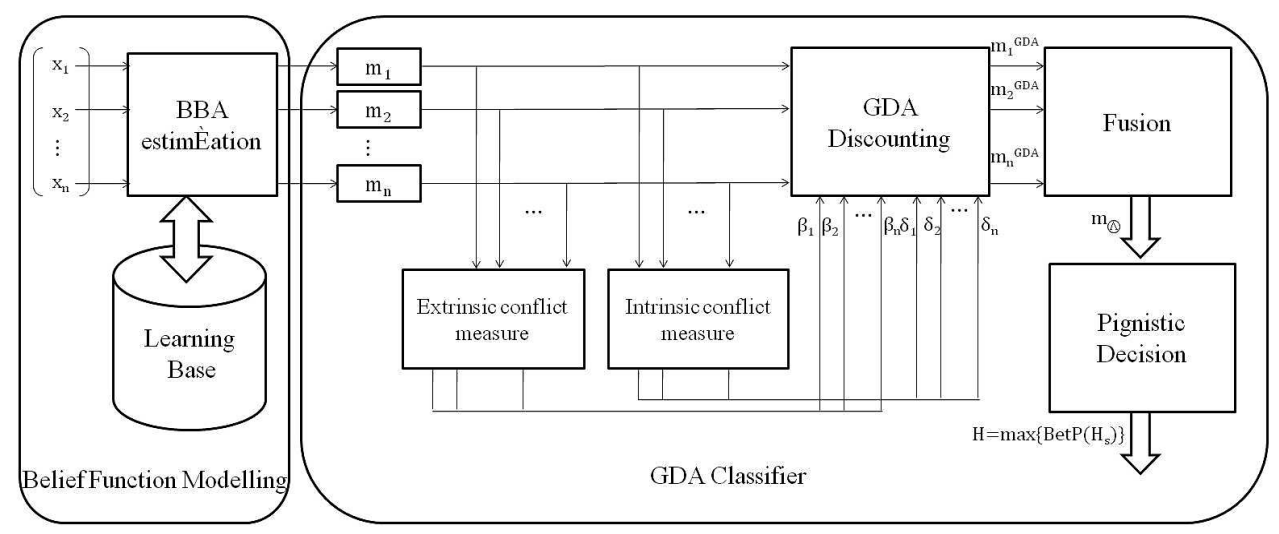

Fig. 2. The proposed GDA classifier architecture

$$
\left\{\begin{array}{l}
m_{i}\left(\left\{H_{n}\right\}\right)=\alpha^{i} \phi^{i}\left(d^{i}\right) \\
m_{i}(\Omega)=1-\alpha^{i} \phi^{i}\left(d^{i}\right)
\end{array}\right.
$$

where $0<\alpha^{i}<1$ is a constant. $\phi^{i}($.$) is a decreasing function fulfilling \phi^{i}(0)=1$ and $\lim _{d \rightarrow \infty} \phi^{i}(d)=0, d_{i}$ is the Euclidean distance between the vector $x$ and the $i$-th prototype. The $\phi^{i}$ function might be an exponential function following this form:

$$
\phi^{s}\left(d^{s}\right)=\exp \left(-\gamma^{s}\left(d^{s}\right)^{2}\right)
$$

where $\gamma^{s}$ is a positive parameter associated to a prototype $s$ and $d^{s}$ is the distance between prototype $s$ and $x$. A learning algorithm was proposed by ${ }^{38}$ to determine the parameters $\gamma^{s}$ in the equation (38) by optimizing an error criterion. The constructed BBAs are then fused following the Dempster rule of combination as follows:

$$
m=\oplus_{i \in[1, \ldots, I]} m_{i}
$$

The aforementioned classification approach is the mono-dimensional variant of the distance classifier. In addition, the multi-dimensional strategy consists in modelling the information according to every characteristic $x_{j}$ (with $j \in[1: J]$ ) of the vector $x$ to classify. The expression of equation 37 becomes:

$$
\left\{\begin{array}{l}
m_{i j}\left(\left\{H_{n}\right\}\right)=\alpha_{j}^{i} \phi_{j}^{i}\left(d_{i j}\right) \\
m_{i j}(\Omega)=1-\alpha_{j}^{i} \phi_{j}^{i}\left(d_{i j}\right)
\end{array}\right.
$$

where $0<\alpha_{j}^{i}<1$ is a constant and $d_{j}^{i}$ represents the distance between the $j$-th component $x_{j}$ of the vector $x$ and its neighbouring vector $v_{i}(i \in[1, K])$. The function $\phi_{j}^{i}$ can be expressed in the following way: 


$$
\phi_{j}^{i}(d)=\exp \left(-\gamma_{i j}\left(d_{i j}^{2}\right)\right.
$$

The use of Dempster's combination operator makes it possible to merge those $K$ belief functions. $m_{j}$ is the resulting belief function and it is equal to:

$$
m_{j}=\oplus_{i \in[1, k]} m_{i j} .
$$

Thanks to its two hypothesis constructed BBA (see equation (40)), this model avoids combinatorial explosion resulting from several fusion processes. Thus, a unique belief function $m$ is obtained by the application of the same fusion principle on those resulting $J$ BBAs:

$$
m=\oplus_{j \in[1, J]} m_{j}
$$

with $J$ the number of sources.

\subsection{The GDA Classifier}

The GDA discounting approach is integrated in the distance classifier in order to prune unreliable sources before the fusion phase. The level of the GDA integration differs following the chosen variant of the distance classifier. For the monodimensional distance classifier, the application of the GDA discounting formula (equation 32 or 36) is applied before the source's fusion (equation 39). However, for the multi-dimensional, the GDA discounting formula is applied after the neighbours fusion (equation 42) and before source's BBA fusion (equation 43). As stated in remark 2, after GDA discounting of the BBAs, the independence of the sources is no longer preserved. In our approach, we used the cautious conjunctive rule (equation 13) after any GDA discounting. The computational complexity of the provided classifier is mainly the complexity of belief function estimation approach. Indeed, for a dataset of $n$ lines and $m$ columns, the computational complexity is equal to $C_{\text {comb }} O(n \times m \times k)$ with $k$ is the fixed value of the sought neighbours. $C_{\text {comb }}$ is the Dempster combination cost.

\section{Experimentation and results}

The experimentation of both GDA approaches were conducted at two stages. In the first stage, the test was carried out on several UCI benchmarks ${ }^{39}$. Indeed, the GDA classifier in its two versions (with GDA-E and GDA-W discounting) was tested in a classification problem comparatively to several pioneer classifiers. In the second stage, we considered an urban image classification problem.

\section{1. dataset classification experiments}

The experimentation of the GDA classifier was carried out on several UCI benchmarks ${ }^{39}$. The characteristics of these datasets are summarized in Table 5 . 
Table 5. dataset characteristics description

\begin{tabular}{|l|r|r|r|}
\hline dataset & \#Instances & \#Attributes & \#Classes \\
\hline Iris & 150 & 4 & 3 \\
\hline Wine & 178 & 13 & 3 \\
\hline ILPD (Indian Liver Patient dataset) & 583 & 10 & 2 \\
\hline Diabetes & 767 & 9 & 2 \\
\hline Image segmentation & 1500 & 20 & 7 \\
\hline
\end{tabular}

We carried out the tests using the mono-dimensional version of GDA classifier. For the classification task, we applied a cross-validation technique.

The results will be compared to several referenced works. Then, we are proposing an oriented discounting conflict management approach. We compare our results to $^{5}$ 's work (described in subsection 3.3 and denoted Mart) which has shown that it outperforms its predecessors. In order to get a general idea of our belief formalism classifier, we analyse the difference between the proposed approach and the Distance Classifier (DC). Since each tested method is based on the $K N N$ algorithm, we fixed $K=4$ for all of them. We also carried out a comparison with the Naive Bayes Classifier (denoted as Bayes in Tables 6). Table 6 shows classification results of tested classifiers where '\#' and '\%' indicate, respectively, the number and the percentage of correct classified instances.

Table 6. Comparative results for datasets' classification

\begin{tabular}{|l|r|r|r|r|r|r|r|r|r|r|r|r|}
\cline { 2 - 12 } \multicolumn{1}{c|}{} & \multicolumn{2}{c|}{ DC } & \multicolumn{2}{c|}{ Mart } & \multicolumn{2}{c|}{ GDA-W } & \multicolumn{2}{c|}{ GDA-E } & \multicolumn{2}{c|}{ KNN } & \multicolumn{2}{c|}{ Bayes } \\
\cline { 2 - 12 } \multicolumn{1}{c|}{} & $\#$ & $\%$ & $\#$ & $\%$ & $\#$ & $\%$ & $\#$ & $\%$ & $\#$ & $\%$ & $\#$ & $\%$ \\
\hline Iris & 145 & 96.67 & 145 & 96.67 & $\mathbf{1 4 6}$ & $\mathbf{9 7 . 3 3}$ & $\mathbf{1 4 6}$ & $\mathbf{9 7 . 3 3}$ & 143 & 95.33 & 144 & 96.00 \\
\hline Wine & 142 & 79.77 & 140 & 78.65 & $\mathbf{1 5 2}$ & $\mathbf{8 5 . 3 9}$ & $\mathbf{1 5 2}$ & $\mathbf{8 5 . 3 9}$ & 169 & 94.94 & $\mathbf{1 7 2}$ & $\mathbf{9 6 . 6 2}$ \\
\hline ILPD & 374 & 64.15 & 378 & 64.83 & $\mathbf{3 8 1}$ & $\mathbf{6 5 . 3 5}$ & $\mathbf{3 8 0}$ & $\mathbf{6 5 . 1 8}$ & 378 & 64.83 & 325 & 55.74 \\
\hline Diabete & 529 & 68.97 & 530 & 69.10 & $\mathbf{5 3 4}$ & $\mathbf{6 9 . 6 2}$ & $\mathbf{5 3 7}$ & $\mathbf{7 0 . 0 1}$ & 539 & 70.18 & $\mathbf{5 8 6}$ & $\mathbf{7 6 . 3 0}$ \\
\hline $\begin{array}{l}\text { Image Seg- } \\
\text { mentation }\end{array}$ & 1400 & 93.33 & 1406 & 93.73 & $\mathbf{1 4 1 8}$ & $\mathbf{9 4 . 5 3}$ & $\mathbf{1 4 0 8}$ & $\mathbf{9 3 . 8 6}$ & $\mathbf{1 4 4 7}$ & $\mathbf{9 6 . 4 6}$ & 1217 & 81.13 \\
\hline
\end{tabular}

As highlighted by statistics shown in Table 6, the proposed approaches allow us to obtain better classification results than the Distance Classifier (DC) for all tested datasets. This fact shows the importance of using a discounting stage before fusion in order to discard unreliable sources. In addition, both classification accuracy are better than flagged out the Martin et al's approach in every treated dataset. Since both classification methods (GDA and Martin et al's approach) differ only in the discounting method, this improvement highlights the importance of using the intrinsic measure to estimate the reliability of a source. We also compared other non based belief function theory classifiers such as KNN and Naive Bayes Classifier. This comparison aims at situating our classifier within some known classical ones. By comparing GDA-E and GDA-W to the $K N N$ classifier, we notice that we have 
also improved the obtained results for the Iris and ILPD datasets. This improvement can be interpreted as the contribution of uncertainty modelling and multi-source fusion. However, for the Wine and image segmentation datasets, the $K N N$ presents a better results. On the other hand, the Naive Bayes Classifier presents a better classification results for Wine and Diabete datasets. This fact proves that Bayesian formalism handles better the uncertainty for those datasets. Nevertheless, for the ILPD dataset the Naive Bayes Classifier results are low.

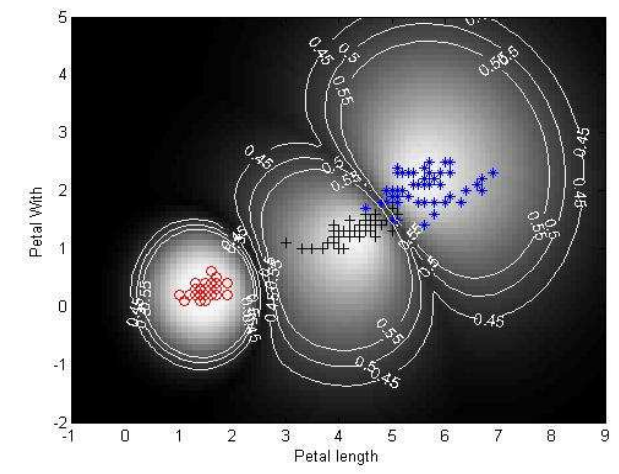

Fig. 3. Pignistic probability maxima for DC approach in Iris dataset.

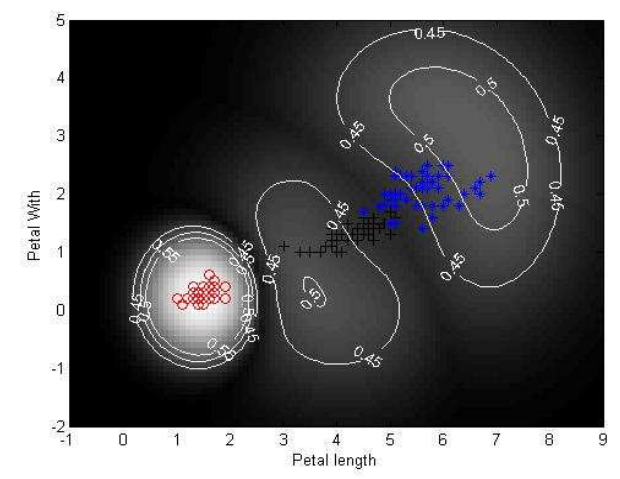

Fig. 5. Pignistic probability maxima for GDA-W approach in Iris dataset $(k=$ $0.5, l=2$ )

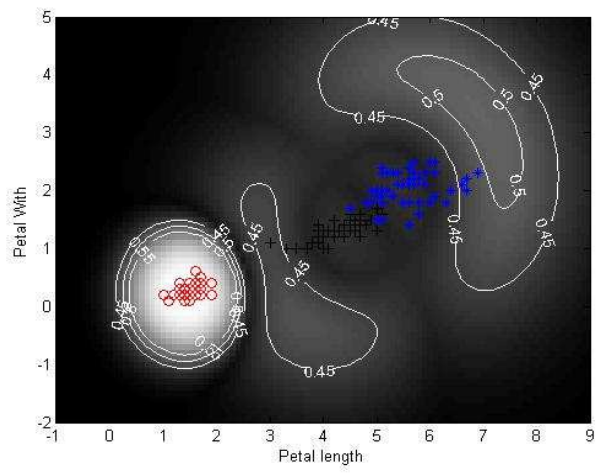

Fig. 4. Pignistic probability maxima for Martin's approach in Iris dataset

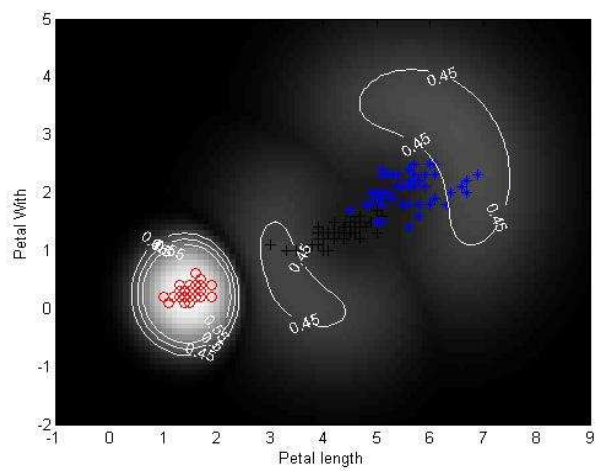

Fig. 6. Pignistic probability maxima for GDA$\mathrm{E}$ approach in Iris dataset

In the following, we compare the belief based classification methods. Figures 3, 4, 5 and 6 , respectively, show the different elements of Iris training base studied according to their petal width and length. In those figures, we illustrate the isopignistic curves where bright zones correspond to a high pignistic probability value 
whereas the dark one indicates the opposite. For Iris-setosa vectors (red colored in Figures), we notice that the pignistic probability is the same for every classification method thanks to the class uniformity of all extracted neighbours. For the two other classes, we noticed significant differences between studied approaches specially in the bordering area. In the DC approach Figure (see Figure 3), the class change is operated roughly leading to classification errors at the decision stage. However, GDA and Martin et al's approaches (Figures 4,5 and 6) present a low pignistic probability in borders. This result is a natural consequence of discounted BBA that contributes to representing better the doubt between both classes. For Martin's and GDA-E discounting the doubt zone is the largest whenever compared to GDA-W which rejects fewer vectors in decision. Nevertheless, even though the reject zone is large for both methods, the GDA-E presents a high pignistic probability value than do Martin et al's approach.

\subsection{Urban image classification experiments}

The GDA classification was also tested on a Quickbird image covering urban areas of Strasbourg, taken in 2008, having four bands, each band has $2.44-2.88 \mathrm{~m} / \mathrm{px}$ as spatial resolution. This image contains a variety of objects: houses, parks, road, etc. Those objects can be reduced to three major concepts. For this reason, in the following, we are mainly interested in finding roads, buildings and vegetation which represent almost all possible objects in the image. Those three classes constitute our frame of discernment $\Omega=\{$ Roads, Building, Vegetation $\}$. In order to extract those classes correctly, we have used five different sources. Some of the used sources correspond to a band from the image and others represent image products. The experiments were conducted on a basis of 8712 pixels. Indeed, we tested our approach on 2256 building pixels, 2926 road pixels and 3530 vegetation pixels. The considered sources are:

- R-G-B

- Near Infrared (NIR)

- Normalized Difference Vegetation Index (NDVI).

The NDVI denotes the vegetation index ${ }^{40}$ and is given by this formula:

$$
N D V I=\frac{N I R-V I S}{N I R+V I S}
$$

where VIS and NIR respectively stand for the radiometry measurements acquired in the visible (red) and near-infrared regions. Each source can identify the considered classes with a certain level of reliability. This fact makes from this context an adequate experimentation field for the GDA discounting approaches. Figure 7 shows the original high-resolution image that we tried to classify.

We compared the conflict rate of our proposed approach with those obtained respectively by conjunctive operator and ${ }^{5}$ discounting approach in order to highlight conflict decrease. 


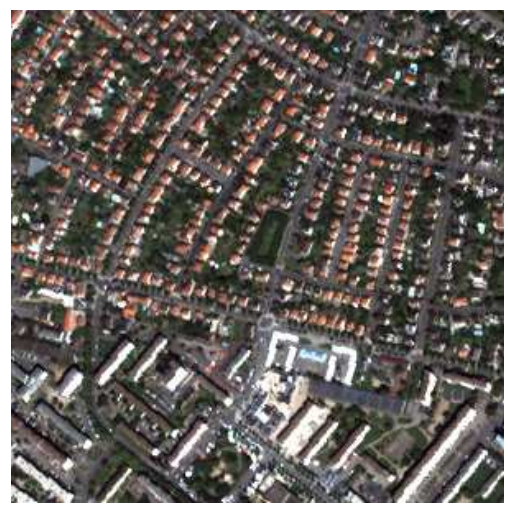

Fig. 7. Original high-resolution image of a Strasbourg site.

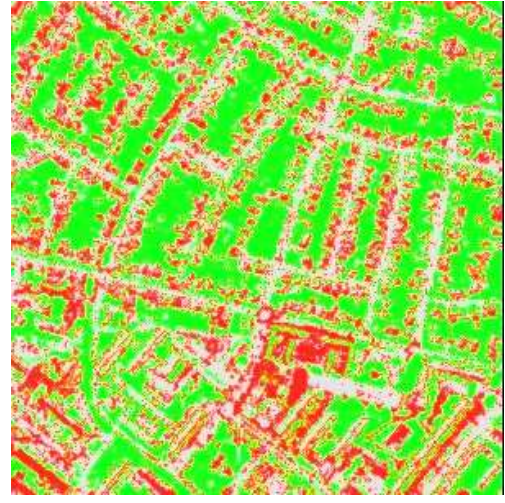

Fig. 8. Classification using GDA-W with only the extrinsic measure $(k=$ $0)$.

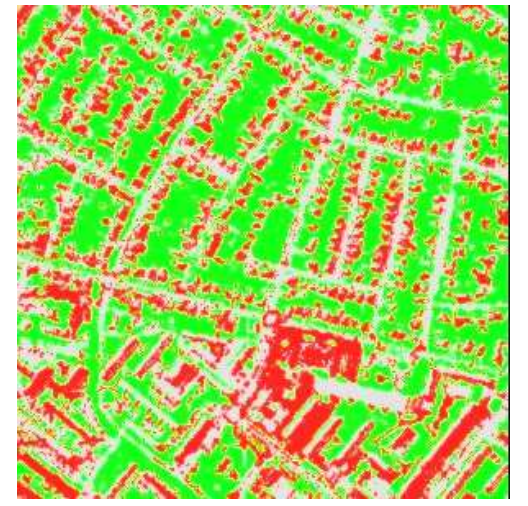

Fig. 9. Classification using GDA-W with the extrinsic and intrinsic measures $(k=0.2$ and $l=1)$.

Figures 8 shows the classification results obtained with the use of only the extrinsic measure, where the vegetation, road, building classes are represented respectively by the colors green, gray and red. The GDA classifier with these settings is denoted the GDA-without and its classification performance are shown in Table 7 . In addition, Figure 9 shows the GDA-W classification results obtained with the integration of both conflict origins. Indeed, the intrinsic conflict rate is an important piece of information about the total reliability of the source. The less the source is confused, the more it is reliable.

The proposed generic discounting is based on two pieces of information: intrinsic and extrinsic conflict measures (equation (34)). The integration of the intrinsic conflict rate for a source $S_{i}$ constitutes the main added value of our method. In order to assess its contribution to classification improvement, we have tried 


\begin{tabular}{|c|c|c|c|c|c|c|c|}
\hline & Building & Road & Vegetation & & Building & Road & Vegetation \\
\hline Building & $70.21 \%$ & $28.41 \%$ & $1.38 \%$ & Building & $65.42 \%$ & $30.98 \%$ & $3.60 \%$ \\
\hline Road & $17.19 \%$ & $82.53 \%$ & $0.28 \%$ & Road & $20.33 \%$ & $79.32 \%$ & $0.35 \%$ \\
\hline Vegetation & $0.00 \%$ & $0.36 \%$ & $99.64 \%$ & Vegetation & $0.00 \%$ & $1.74 \%$ & $98.26 \%$ \\
\hline
\end{tabular}

\begin{tabular}{|c|c|c|c|c|c|c|c|}
\hline & Building & Road & Vegetation & & $\overline{\text { Building }}$ & Road & Vegetation \\
\hline Building & $70.25 \%$ & $28.50 \%$ & $1.25 \%$ & Building & $74.29 \%$ & $25.09 \%$ & $0.62 \%$ \\
\hline Road & $16.50 \%$ & $83.32 \%$ & $0.18 \%$ & Road & $18.73 \%$ & $80.45 \%$ & $0.82 \%$ \\
\hline Vegetation & $0.21 \%$ & $0.15 \%$ & $99.64 \%$ & Vegetation & $0.00 \%$ & $0.07 \%$ & $99.93 \%$ \\
\hline
\end{tabular}

\begin{tabular}{|c|c|c|c|c|c|c|c|}
\hline & Building & Road & Vegetation & & Building & Road & Vegetation \\
\hline Building & $59.26 \%$ & $24.11 \%$ & $16.63 \%$ & Building & 92.40\% & $7.55 \%$ & $0.05 \%$ \\
\hline Road & $21.53 \%$ & $78.23 \%$ & $0.24 \%$ & Road & $5.68 \%$ & $93.97 \%$ & $0.35 \%$ \\
\hline Vegetation & $0.34 \%$ & $2.35 \%$ & $97.31 \%$ & Vegetation & $0.00 \%$ & $0.34 \%$ & $99.66 \%$ \\
\hline
\end{tabular}

\begin{tabular}{|l|r|r|r|}
\cline { 2 - 3 } \multicolumn{1}{c|}{} & Building & Road & Vegetation \\
\hline Building & $\mathbf{7 9 . 5 1 \%}$ & $20.49 \%$ & $0.00 \%$ \\
\hline Road & $4.34 \%$ & $\mathbf{9 3 . 8 0 \%}$ & $1.86 \%$ \\
\hline Vegetation & $0.12 \%$ & $0.14 \%$ & $\mathbf{9 9 . 7 4 \%}$ \\
\hline \multicolumn{4}{|c|}{ Bayes }
\end{tabular}

Table 7. Confusion matrices

to compare the classification results obtained with only the extrinsic information (denoted GDA-without) to the classifier integrating both conflict information (GDA-E and GDA-W). In addition, we compared ourselves to the $K N N$ and the Naive Bayes Classifier (denoted Bayes).

Table 7 illustrates the confusion matrices of tested classification approaches for buildings, roads and vegetation detection. The matrices also highlight the intrinsic measure integration contribution. Indeed, by comparing the GDA-W and GDA-E to GDA-without, all considered classes detection was improved with different proportions. The best improvement was the building class which means that fused information sources were confused when we tried to classify them. In this case, the GDA-E and GDA-W consider discount the sources following their internal and external conflicts. Furthermore, road and vegetation class detection was slightly improved relatively to the building class. Nevertheless, the provided results were high. Indeed, since each source is specialized in detecting specific classes, the introduction of the GDA allowed us to avoid conflict generation by discounting those unreliable sources. The improvement of detection of all classes (comparatively to 
GDA-without) illustrates the compromises in terms of reliability that GDA seeks to find between source each time a pixel is under classification. For example, whenever handling a road pixel, the NDVI source (specialized in vegetation detection) is highly discounted since its assigned BBA is confused.

We also conducted comparative experiments to other based belief function works such $a^{5}$ discounting approach. We also compared our results to the Distance Classifier (DC) based on distance BBA estimation (see subsection 5.1) and pignistic decision (see subsection 2.5). As is shown in Table 7, comparatively to the DC approach, we sharply improved the results for each class. We have also improved Martin et al's result for the road class with the GDA-W discounting and we maintained the same average for the vegetation. Interestingly enough, the GDA-E provides the best results for the building and the vegetation recognition considering all belief based classification approaches. In order to position our work in terms of pure classification, we compared our results to non belief based known classifiers such as $K N N$ and NaiveBayes. In general, KNN provides the best classification results for building and road detection. For the vegetation class all classifiers provide almost the same good classification rates with a slight advantage for the GDA-E. Despite the performance difference between our approaches and the $K N N$ in this particular context of application, comparison can not be made directly since they rely on different formalism. In addition, we do provide the best results comparatively to belief classifiers and discounting based approaches.

Figures 11, 12, 13 and 14 illustrate the classification of an urban site (Figure 10) with respectively the proposed DC, Martin, GDA-W and GDA-E classification approaches. We can notice that in this sample we improved through our approach the extraction of the road and building classes.

The figure 15 and 16 show the pixels (i.e, belief functions) where we registered an important conflict rate and the GDA approaches changed their initial classification (DC classification approach).

\section{Conclusion}

In this work, we introduced a conflict management approach named GDA. It allows to discount any information source following its estimated reliability. We introduced a new measure of reliability based on two conflict origins: Intrinsic and Extrinsic conflict. The discounting approach, proposed in two different versions GDA-W and GDA-E, was integrated into a based distance belief function classifier. The GDA approach was thoroughly experimented on several UCI datasets and on urban image classification problem. The provided results confirm the contribution of both conflict measures association. Even though very encouraging results were obtained, comparatively with other discounting approaches, we aim to improve the introduced approach in further works by studying other conflict measures association. Additionally, further conflict origins can be studied like lying and insincere sources. Image classification improvement could be investigated by studying other 


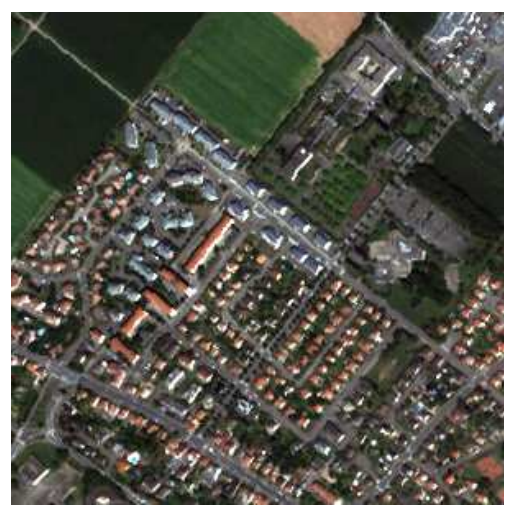

Fig. 10. Original image.

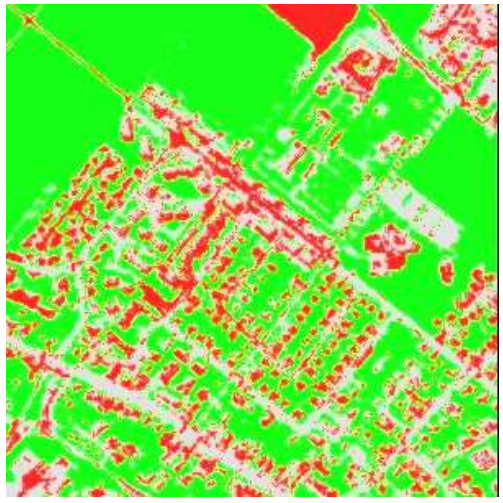

Fig. 12. Classification using Martin et al's discounting approach.

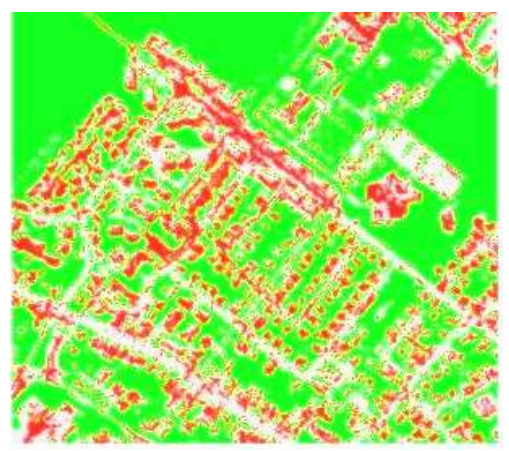

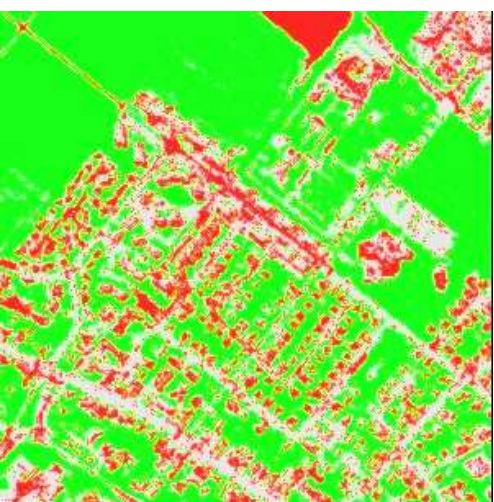

Fig. 11. Classification using DC approach.

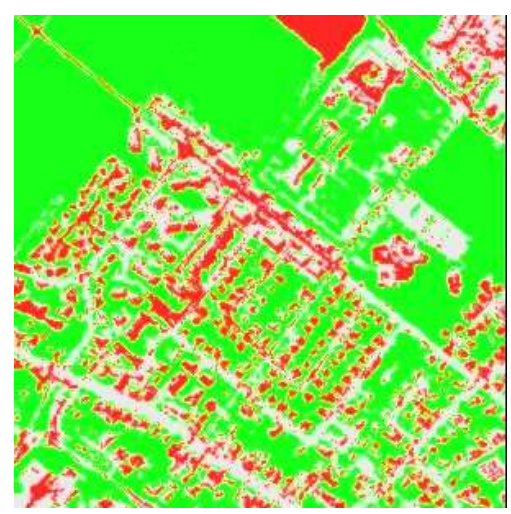

Fig. 13. Proposed GDA-W classification.

Fig. 14. Classification using GDA-E discounting approach.

approaches. In this work, we considered a pixel classification but in future work we plan to consider a region based approach. Indeed, a region based approach can 


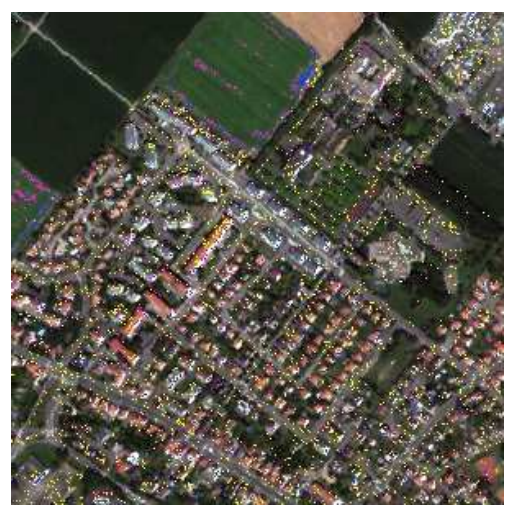

Fig. 15. Pixels where GDA-E approach changed the initial classification.

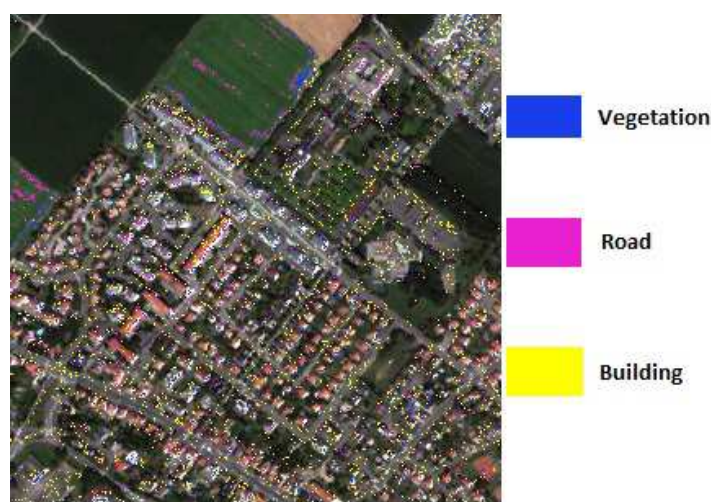

Fig. 16. Pixels where GDA-W approach changed the initial classification.

provide valuable information such as: dimension, shape, etc.

\section{Acknowledgements}

The authors would like to express their sincere gratitude to Sebastien Destercke CNRS researcher at Heudiasyc laboratory for his advices and his feedbacks. Thanks are also due to the anonymous reviewers for their constructive and helpful comments and suggestions which have been in help to improve the quality of this paper.

\section{References}

1. A. Dempster, Upper and lower probabilities induced by multivalued mapping, AMS$38,1967$.

2. G. Shafer, A Mathematical Theory of Evidence, Princeton University Press, Princeton, 1976.

3. L. A. Zadeh, A simple view of the Dempster-Shafer theory of evidence and its implicationfor the rule of combination, The AI Magazine 7, no. 2 (1994) 85-90.

4. J. Klein, O. Colot, Automatic discounting rate computation using a dissent criterion, in Proceedings of the Workshop on the Theory of Belief Functions, Brest, France (2010) 1-6.

5. A. Martin, A.-L. Jousselme, C. Osswald, Conflict measure for the discounting operation on belief functions, in Proceedings of 11th International Conference on Information Fusion, Cologne, Germany (2008) 1003-1010.

6. J. Schubert, Conflict management in Dempster-Shafer theory using the degree of falsity, International Journal of Approximate Reasoning 52 (3) (2011) 449-460.

7. K. Sentz, S. Ferson, Combination of evidence in Dempster-Shafer theory, Tech. rep. (2002).

8. P. Smets, Analyzing the combination of conflicting belief functions, Information Fusion 8 (4) (2007) 387-412.

9. H. Guo, W. Shi, Y. Deng, Evaluating sensor reliability in classification problems based on evidencetheory, IEEE transactions on Systems, Man, and Cybernetics, Part B 36 (5) (2006) 970-981. 
10. A.-L. Jousselme, D. Grenier, E. Bossé, A new distance between two bodies of evidence, Information Fusion 2 (2001) 91-101.

11. M. Daniel, Conflicts within and between belief functions, in Proceedings of the International Conference on Information Processing and Management of Uncertainty, IPMU'10, Dortmund, Germany (2010) 696-705.

12. F. Smarandache, A. Martin, C. Osswald, Contradiction measures and specificity degrees of basic belief assignments, in Proceedings of International Conference on Information Fusion, Chicago, Illinois (2011) 1-8.

13. W. Liu, Analyzing the degree of conflict among belief functions, Artificial Intelligence 170 (2006) 909-924.

14. T. Denoeux, K-nearest neighbor classification rule based on Dempster-Shafer theory, IEEE Transactions on Systems, Man and Cybernetics 25 (5) (1995) 804-813.

15. P. Smets, R. Kennes, The Transferable Belief Model, Artificial Intelligence 66 (2) (1994) 191-234.

16. P. Smets, The combination of evidence in the Transferable Belief Model, IEEE Transactions on Pattern Analysis and Machine Intelligence 12 (5) (1990) 447-458.

17. T. Denœux, Conjunctive and disjunctive combination of belief functions induced by nondistinct bodies of evidence, Artificial Intelligence 172 (2) (2008) 234-264.

18. P. Smets, Decision making in the TBM : The necessity of the pignistic transformation, International Journal of Approximate Reasoning 38 (2005) 133-147.

19. J. Klein, O. Colot, Singular sources mining using evidential conflict analysis, International Journal of Approximate Reasoning 52 (9) (2011) 1433-1451.

20. A. Martin, About conflict in the theory of belief functions, in Proceedings of International Conference on Belief Functions, BELIEF'2012, Compiègne, France (2012) 161-168.

21. S. Destercke, T. Burger, Toward an axiomatic definition of conflict between belief functions, IEEE Systems, Man and Cybernetics - part B 43 (2) (2012) 585-596.

22. P. Smets, Analyzing the combination of conflicting belief functions, Information Fusion 8 (4) (2007) 387-412.

23. F. Cuzzolin, A geometric approach to the theory of evidence, IEEE transaction, Man, and Cybernetics-Part C: Application and reviews 38 (4) (2008) 522-534.

24. B. Tessem, Approximations for efficient computation in the theory of evidence, Artificial Intelligence (1993) 315-329.

25. A.-L. Jousselme, P. Maupin, Distance in evidence theory: Comprehensive survey and generalizations, International Journal of Approximate Reasoning 53 (2) (2012) 118145.

26. T. Burger, Geometric interpretations of conflict: A viewpoint, In Proceedings of 3rd International Conference on Belief Functions, Belief 2014, Oxford, UK 8764 (2014) 412-421.

27. P. Smets, The application of the matrix calculus to belief functions, International Journal of Approximate Reasoning 31 (12) (2002) 1 - 30.

28. T. George, N. R. Pal, Quantification of conflict in Dempster-Shafer framework: A new approach, International Journal of General Systems 24 (4) (1996) 407-423.

29. R. R. Yager, On considerations of credibility of evidence, International Journal of Approximate Reasoning 7 (1992) 45-72.

30. Z. Elouedi, K. Mellouli, P. Smets, Assessing sensor realibility for multisensor data fusion within the Tranferable Belief Model, IEEE Transaction on System, Man and Cybernetics - Part B 34 (1) (2004) 782-787.

31. Z. Elouedi, E. Lefevre, D. Mercier, Discounting of a belief function using a confusion matrix, in Proceedings of the 22th IEEE International Conference on Tools with 
Artificial Intelligence, ICTAI'2010, Arras, France (2010) 287-294.

32. Y. Deng, W. Shi, Z. Zhu, Q. Liu, Combining belief functions based on distance of evidence, Decision Support Systems 38 (3) (2004) 489-493.

33. L. Chen, W. Shi, Y. Deng, Z. Zhu, A new fusion approach based on distance of evidences, Journal of Zhejian University Science 6A (2005) 476-482.

34. Z.-G. Liu, Q. Pan, Y.-M. Cheng, J. Dezert, Sequential adaptative combination of unreliable sources of evidence, in Proceedings of Workshop on the Theory of Belief Function, Brest, France (2010) Paper no 89.

35. A. Samet, I. Hammami, E. Lefevre, A. Hamouda, Generic discounting evaluation approach for urban image classification, In Proceedings of 3rd international symposium on Integrated Uncertainty in Knowledge Modelling and Decision Making, IUKM'2013, Beijing, China (2013) 79-90.

36. Z. Elouedi, K. Mellouli, P. Smets, Belief decision trees: theoretical foundations, Int. J. Approx. Reasoning 28 (2-3) (2001) 91-124.

37. P. Vannoorenberge, T. Denoeux, Likelihood-based vs. distance-based evidential classifiers, In Proceedings of International Conference on Fuzzy Systems FUZZ-IEEE, Melbourne, Australia (2001) 320 - 323 .

38. L. Zouhal, T. Denoeux, An evidence-theoretic K-NN rule with parameter optimization, IEEE Transactions on Systems, Man and Cybernetics, Part C 28 (2) (1998) 263-271.

39. A. Frank, A. Asuncion, UCI Machine Learning Repository http://archive.ics.uci.edu/ml (2010).

40. R. E. Crippen, Calculating the vegetation index faster, Remote Sensing of Environment 34 (1) (1990) $71-73$.

\section{Biographical Sketch and Photo}

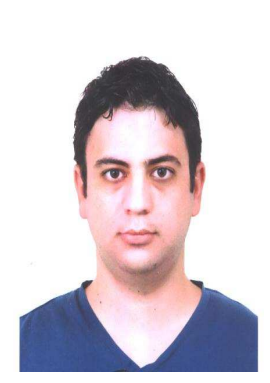

Ahmed Samet received the M.Sc. degree in Computer Science from Faculty of Sciences of Tunis (Tunisia) in 2010, the Ph.D. in Computer Science from the Faculty of Sciences of Tunis (Tunisia), and Univeristé d'Artois (France). Currently, he is a postdoctoral researcher with Sorbonne University, Université de technologie de Compiègne (France).

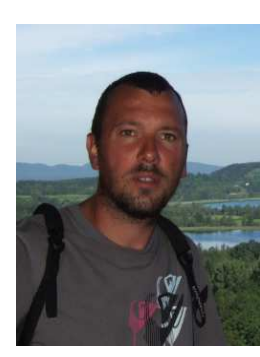

Eric Lefevre received, in 2001, the Ph.D. in physics from the Institut National des Sciences Appliquées (INSA) in Rouen. He joined the Univeristé d'Artois in 2002 , as associate professor. He obtained the "Habilitation à diriger des recherches" in 2012 and, as of 2013, he is full professor. His research interests include uncertainty modeling and information fusion. 


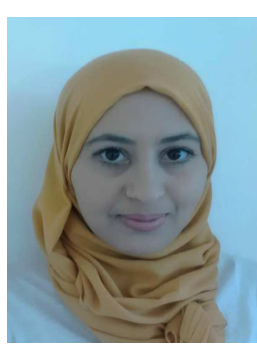

Imen Hammami received the M.Sc. degree in Computer Science from Faculty of Sciences of Tunis (Tunisia) in 2012. Currently, she is a Ph.D. student in Computer Science with the Faculty of Sciences of Tunis (Tunisia) and TELECOM Bretagne, Département Image et Traitement Information (France).

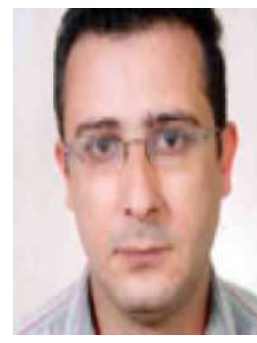

Sadok Ben Yahia received the M.Sc. degree in Computer Science from ENSI High School, Tunisia in 1996, the Ph.D. in Computer Science from the Faculty of Sciences of Tunis (University Tunis ElManar), and the Habilitation to Lead Researches Diploma from the University of Montpellier (France). Currently, he is a full professor with Faculty of Sciences of Tunis, Tunis, Tunisia. 